\title{
The emerging role of local therapy in oligometastatic non-small cell lung cancer
}

\author{
James M. Isbell, MD, MSCI, ${ }^{\mathrm{a}, \mathrm{b}}$ Bob T. Li, MD, PhD, MPH, ${ }^{\mathrm{b}, \mathrm{c}}$ and Daniel R. Gomez, MD, MBA ${ }^{\mathrm{b}, \mathrm{d}}$
}

Feature Editor Introduction-Local therapy for oligometastatic non-small cell lung cancer (NSCLC) is a complex and controversial topic that continues to evolve. With advances in systemic targeted agents, immunotherapy, and imaging modalities, thoracic surgeons are being asked to consider resection in patients with advanced lung cancer with a limited number of metastases with curative intent. In this Invited Expert Opinion article, Isbell and colleagues, including co-authors in medical and radiation oncology, provide an excellent overview of data supporting the existence of an oligometastatic state and recent studies on local consolidative therapy with surgical metastasectomy and stereotactic ablative radiotherapy. Most surgery studies are retrospective, and randomized trials are lacking. Most of the recent data in this area focus on treatment with stereotactic radiation, including 3 recent phase 2 prospective trials suggesting that local therapy prolongs overall and progression-free survival, although the Stereotactic Ablative Radiotherapy for the Comprehensive Treatment of Oligometastatic Disease trial included tumors other than NSCLC. Dr Gomez, one of the co-authors of the current article, is the lead author on one of these published phase 2 trials. With this growing evidence, several international guidelines now recommend local therapy for selected patients with oligometastatic disease. While we await the results of several ongoing randomized trials, including the OMEGA, NRG-LU002, and SARON trials, the authors conclude that local treatment of oligometastatic NSCLC with surgery or ablative therapies can provide survival benefit in carefully selected patients who respond to firstline systemic therapy.

\section{Jules Lin, MD}

Thoracic surgeons are increasingly asked to consider surgical resection for patients with advanced non-small cell lung

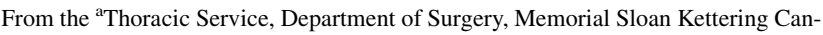
cer Center, New York, NY; ${ }^{\mathrm{b}}$ Weill Cornell Medicine, New York, NY; ${ }^{\mathrm{c}}$ Thoracic Oncology Service, Division of Solid Tumor Oncology, Department of Medicine, Memorial Sloan Kettering Cancer Center, New York, NY; and ${ }^{\mathrm{d}}$ Thoracic Radiation Oncology Service, Department of Radiation Oncology, Memorial Sloan Kettering Cancer Center, New York, NY.

This work was supported in part by the National Institutes of Health Comprehensive Cancer Center Support Grant (P30 CA008748).

Received for publication Nov 3, 2020; revisions received March 1, 2021; accepted for publication April 9, 2021; available ahead of print May 29, 2021.

Address for reprints: James M. Isbell, MD, MSCI, Thoracic Service, Department of Surgery, Memorial Sloan Kettering Cancer Center, 1275 York Ave, Box 7, New York, NY 10065 (E-mail: isbellj@mskcc.org).

J Thorac Cardiovasc Surg 2022;163:819-25 $0022-5223 / \$ 36.00$

Copyright (c) 2021 Published by Elsevier Inc. on behalf of The American Association for Thoracic Surgery

https://doi.org/10.1016/j.jtcvs.2021.04.090
}

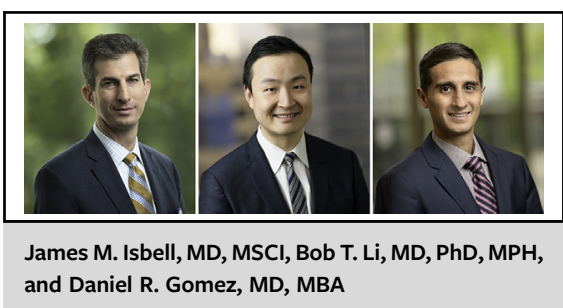

CENTRAL MESSAGE

In carefully selected patients, local treatment of oligometastatic NSCLC with surgical resection or ablative therapies can provide a substantial survival benefit.

See Commentary on page 826.

cancer (NSCLC) with a limited number of metastases. Driving this apparent increased incidence of oligometastatic disease may be improvements in the efficacy of systemic therapies and enhanced detection of metastases with modern imaging. Even before the widespread adoption of checkpoint inhibitors and targeted therapies for metastatic NSCLC, studies demonstrated 5-year overall survival (OS) of $29 \%$ to $45 \%$ for patients with limited metastases who underwent surgery or ablation to all disease sites. ${ }^{1-3}$ The added success of newer cytotoxic, targeted, and immune therapies in controlling systemic disease has further expanded the pool of patients with oligometastatic NSCLC and, consequently, increased the use of potentially curative, rather than merely palliative, treatment strategies for these patients.

\section{DEFINITION OF OLIGOMETASTATIC DISEASE}

Hellman and Weichselbaum ${ }^{4}$ first proposed the concept of oligometastasis, describing it as an intermediate state between localized cancer and widely metastatic disease. Although the definition of oligometastatic NSCLC varies considerably, it generally refers to metastatic disease limited to 5 or less sites with a more indolent tumor biology. There are multiple oligometastatic disease states that may be classified by the timing of their initial detection and response to therapy, as summarized in Table 1.

Two recent consensus papers have attempted to define oligometastasis in a more nuanced manner-the first by 
the European Organisation for Research and Treatment of Cancer and the European Society for Radiotherapy and Oncology. ${ }^{6}$ The second consensus statement was a collaboration between European Organisation for Research and Treatment of Cancer and the European Society for Radiotherapy and Oncology and the American Society for Radiation Oncology. ${ }^{7}$ Details of these consensus statements are beyond the scope of this review, but the interested reader is referred to them for further guidance on detailed consensus definitions for oligometastatic disease.

\section{EVIDENCE FOR AN OLIGOMETASTATIC STATE}

The evidence for an oligometastatic state in NSCLC is growing. In a systematic review querying the oligometastatic state in NSCLC, Ashworth and colleagues ${ }^{1}$ identified 49 eligible studies and 2176 patients. Eligible patients had 1 to 5 metastases treated with surgical metastasectomy, stereotactic ablative radiotherapy (SABR), or stereotactic radiosurgery (SRS). Sixty percent of the studies included patients with brain metastases only. The primary tumor was controlled in $82 \%$ of patients. Median OS was 14.8 months for all patients (range, 5.9-52). Among patients with a controlled primary tumor, median OS was 19 months. The overall median time to any progression was 12 months. Multivariable analysis showed that definitive treatment of the primary tumor, $\mathrm{N}$-stage, and a disease-free interval of at least 6 to 12 months were statistically significant prognostic indicators.

In a follow-up study, the authors performed an individual-patient meta-analysis to evaluate outcomes and prognostic factors after treatment for oligometastatic NSCLC. ${ }^{2}$ They obtained patient-level data on 757 patients with oligometastatic NSCLC (1-5 synchronous or metachronous metastases) who underwent curative treatment of the primary lung cancer along with surgical metastectomy, SABR or SRS, or radical external-beam radiotherapy to address the metastatic sites. Predictors of OS and progression-free survival (PFS) were identified using Cox proportional hazards regression models. Median OS was 26 months; 5-year OS was $29 \%$. Surgery was the most common treatment for both the primary tumor $(84 \%)$ and metastases $(62 \%)$. Predictors of improved OS included metachronous over synchronous metastases $(P<.001)$, lower $\mathrm{N}$-stage $(P<.002)$, and adenocarcinoma histologic subtype $(P<.036)$. Recursive partitioning analysis identified a low-risk group comprising metachronous metastases (5-year OS, 47.8\%), an intermediate-risk group comprising synchronous metastases and NO disease (5-year OS, $36.2 \%$ ), and a high-risk group comprising synchronous metastases and N1/N2 disease (5-year OS, 13.8\%).

Further supporting the existence of an oligometastatic state are the results of a study evaluating patterns of treatment failure after first-line systemic therapy. ${ }^{8}$ Although limited by its small sample size and retrospective design, the study demonstrated that, in $64 \%$ of patients, disease progression was confined to the sites known to exist at initial presentation, suggesting that local therapy to primary and metastatic sites may remove sources of future metastatic spread.

The presence of an oligometastatic state in NSCLC was recognized in the $8^{\text {th }}$ edition of the American Joint Committee on Cancer Staging Manual by further subclassification of the TNM M-stage into M1a, M1b, and M1c. ${ }^{9,10}$ The M1a subclassification, which preserved its definition from the $7^{\text {th }}$ edition, includes (a) pleural/pericardial effusion, (b) contralateral/bilateral tumor nodules, and (c) pleural/ pericardial nodules. Survival analyses comparing patients with a solitary metastasis involving a single organ site to patients with M1a disease or patients with either multiple metastases in a single organ or multiple metastases in more than 1 organ showed a statistically significant difference across these groups. In particular, there was an appreciable difference in survival between patients with a solitary metastasis and patients with multiple metastases (median OS, $11.4[95 \%$ confidence interval $\{\mathrm{CI}\}, 9.6-13.7]$ vs 6.3 [95\% CI, 4.8-7]). Therefore, the M1b subclassification was redefined to include only patients with a solitary metastasis, and a new M1c subclassification was created to include any patients with more than 1 metastasis, regardless of whether a single organ or multiple organs were involved. Of note, among patients with only a single metastatic site, further analysis found that metastatic involvement of any specific organ system (eg, adrenals, brain, bone, liver, skin, or distant lymph node) was not associated with prognosis. ${ }^{9}$ Put another way, in patients with a single metastasis, the metastatic site does not appear to influence survival.

Taken together, the findings of these studies suggest that there is an oligometastatic state in NSCLC and that select

TABLE 1. Definitions of common oligometastatic disease states

\begin{tabular}{ll}
\hline Oligometastatic disease state & \multicolumn{1}{c}{ Definition } \\
\hline Synchronous & Limited number of metastases identified within 6 mo of diagnosis \\
Metachronous $_{\text {Induced }^{5}}$ & Oligometastatic disease manifesting $>6$ mo after resection or ablation of primary tumor (with primary site controlled) \\
Oligoprogressive* $^{*}$ & Polymetastatic disease reduced to an oligometastatic state due to profound response to systemic therapy \\
\hline Oligopersistent & Disease progression in only a few sites while on active systemic therapy \\
\hline
\end{tabular}

*These oligoprogressive sites of disease are thought to arise from the emergence of resistant clones no longer responsive to systemic therapy. 
patients, despite having stage IV disease, can experience long-term survival, potentially even cure, with surgery or ablative therapies to all metastatic sites, arguing against the therapeutic nihilism that sometimes accompanies such cases.

\section{EVIDENCE FOR SURGICAL RESECTION AND ABLATION OF OLIGOMETASTASES}

Until recently, with a couple exceptions, ${ }^{3,11}$ most studies evaluating the efficacy of local consolidative therapies (LCTs; surgery or ablation) for oligometastases have been retrospective. Consequently, appropriate skepticism has been applied to the interpretation of these studies. With the recent publication of 2 phase II randomized controlled trials specific to oligometastatic NSCLC, ${ }^{12-14}$ and one with mixed histologic subtypes including $\mathrm{NSCLC}^{15}$ we now have compelling level 1 evidence that LCT prolongs OS and PFS in patients with oligometastatic NSCLC (Table 2).

The first of these trials to be published was a multicenter phase II randomized controlled trial that included patients with stage IV NSCLC with 3 or less metastatic sites after receiving standard first-line systemic therapy with no disease progression at randomization. ${ }^{12}$ Patients were randomly assigned to the investigational arm (LCT [radiotherapy \pm chemotherapy or surgical resection of all lesions] with or without subsequent maintenance therapy) or control arm (maintenance therapy alone or observation). Because of a statistically significant difference in efficacy between the arms, the Data Safety Monitoring Committee terminated the trial early, after only 49 of a planned 94 patients had been enrolled. Median follow-up for all patients was 12.4 months (interquartile range, 5.5-20.3). Median PFS in the investigational arm was 11.9 months $(90 \% \mathrm{CI}$, 5.7-20.9), compared with 3.9 months ( $90 \%$ CI, 2.3-6.6) in the control arm (hazard ratio, 0.35 [90\% CI, 0.18-0.66]; $\log$-rank $P=.005$ ). The time to appearance of a new metastasis was longer in the investigational arm (median, 11.9 months [90\% CI, 5.7 to not reached] vs 5.7 months
[3.1-7.0]; $P=.0497)$. Adverse events were similar between groups, with no grade 4 events or deaths due to treatment in either. Notably, $28 \%$ of patients underwent surgery to address their primary or metastatic sites of disease, most often in combination with radiotherapy.

Long-term follow-up was recently reported, showing an increased median PFS to 14.2 months in the investigational arm versus 4.4 months in the control arm $(P=.022) .{ }^{13}$ In addition, median OS was 41.2 months in the investigational arm versus only 17.0 months in the control arm $(P=.017)$. Interestingly, in contrast to previous retrospective studies, ${ }^{2,16,17}$ nodal stage (N0/N1 vs N2/N3) was not a predictor of survival (hazard ratio, $1.33 ; P=.44$ ). ${ }^{13}$ Considering the early termination of the study and consequent smaller sample size, there may have been insufficient power to detect a survival difference based on $\mathrm{N}$-stage.

This study also demonstrated that survival after progression was longer in the investigational arm (37.6 vs 9.4 months; $P=.034$ ). The increased time to appearance of a new metastasis and the improved survival after progression in the investigational arm suggest that LCT may be altering the natural course of the disease, possibly by eliminating sources of future metastatic dissemination (eg, chemotherapy-resistant clones), by potentiating the effects of systemic therapy or perhaps by enhancing the body's antitumor immune response via the so-called abscopal effect. ${ }^{18,19}$ Radiation is known to have both immunosuppressive and immunostimulatory effects. The immunostimulatory effects of radiation, such as those caused by the release of tumor antigens with radiation-induced tumor cell death, may contribute to a systemic, immune-mediated antitumor response. However, such abscopal responses are rare. To enhance the abscopal effect, some have proposed adding immune checkpoint inhibitor therapy to radiation in stage IV NSCLC. ${ }^{20}$ These are areas of ongoing preclinical and clinical investigation.

A single-institution phase II randomized controlled trial by Iyengar and colleagues ${ }^{14}$ corroborated the findings of

TABLE 2. Completed randomized controlled trials evaluating local therapy for oligometastatic disease

\begin{tabular}{|c|c|c|c|c|c|c|c|c|}
\hline Study & $\begin{array}{c}\text { Enrolled } \\
\text { subjects, no. }\end{array}$ & $\begin{array}{l}\text { Tumor } \\
\text { type(s) }\end{array}$ & $\begin{array}{c}\text { Metastases, } \\
\text { no. }\end{array}$ & $\begin{array}{c}\text { Systemic } \\
\text { therapy } \\
\text { required } \\
\text { before } \mathrm{LCT} \text { ? }\end{array}$ & $\begin{array}{l}\text { Type of LCT } \\
\text { (\% surgery) }\end{array}$ & $\begin{array}{c}\text { Median } \\
\text { follow-up, mo }\end{array}$ & $\begin{array}{c}\text { Median PFS, } \\
\text { LCT vs } \\
\text { control, mo }\end{array}$ & $\begin{array}{l}\text { Median OS, } \\
\text { LCT vs } \\
\text { control, mo }\end{array}$ \\
\hline $\begin{array}{c}\text { Gomez and } \\
\text { colleagues }\end{array}$ & $49 *$ & NSCLC & $1-3$ & Yes & RT, surgery $(28 \%)$ & 38.8 & 14.2 vs 4.4 & 41.2 vs 17.0 \\
\hline $\begin{array}{l}\text { Iyengar and } \\
\text { colleagues }\end{array}$ & $29 *$ & NSCLC & $1-5$ & Yes & RT & 9.6 & 9.7 vs 3.5 & NA \\
\hline $\begin{array}{l}\text { Palma and } \\
\text { colleagues } \\
\text { (SABR-COMET) }\end{array}$ & 99 & $\begin{array}{l}\text { Lung, breast, } \\
\text { prostate, } \\
\text { CRC, other }\end{array}$ & $1-5$ & No & RT & 51 & 11.6 vs 5.4 & 50.0 vs 28 \\
\hline
\end{tabular}

$L C T$, Local consolidative therapy; $P F S$, progression-free survival; $O S$, overall survival; $N S C L C$, non-small cell lung cancer; $R T$, radiotherapy; $N A$, not available; $S A B R$-COMET, Stereotactic Ablative Radiotherapy for the Comprehensive Treatment of Oligometastatic Disease. *Recruitment was stopped early because of findings of the interim analysis. 
Gomez and colleagues. ${ }^{12}$ The study by Iyengar and colleagues randomized patients with stage IV NSCLC with 5 or less metastatic sites to either SABR plus maintenance systemic therapy or maintenance therapy alone after completion of 4 to 6 cycles of first-line platinum-based chemotherapy without progression. A total of 29 patients were enrolled (SABR plus maintenance chemotherapy, $\mathrm{n}=15$; maintenance chemotherapy alone, $\mathrm{n}=14$ ). The trial was terminated early on the basis of an unplanned interim analysis that demonstrated a significant improvement in PFS in the SABR plus maintenance chemotherapy arm (9.7 vs 3.5 months; $P=.01$ ).

Both the studies by Gomez and colleagues ${ }^{12}$ and Iyengar and colleagues ${ }^{14}$ required standard first-line systemic therapy without disease progression before randomization. Response to first-line therapy may be a key prognostic factor to identify before considering LCT for patients with oligometastatic NSCLC, as highlighted by a single-arm phase II prospective trial conducted by De Ruysscher and colleagues. $^{21}$ In this study, patients with pathologically confirmed stage IV NSCLC with 5 or less metastases at primary diagnosis were eligible, provided all tumor sites were suitable for complete surgical resection or definitive radiotherapy. Entry into this study, in contradistinction to the studies by Gomez and colleagues ${ }^{12}$ and Iyengar and colleagues, ${ }^{14}$ was permitted regardless of response to firstline systemic therapy. Median OS in the study by Gomez and colleagues ${ }^{13}$ was more than 3 -fold that in the study by De Ruysscher and colleagues ${ }^{21}$ (41.2 vs 13.5 months). However, the difference in median PFS was less pronounced (14.2 vs 12.1 months). OS after LCT may be influenced by response to first-line chemotherapy, suggesting that surgery or ablative therapy to oligometastatic sites should be reserved for patients with no evidence of progression after completion of standard first-line systemic therapy.

The Stereotactic Ablative Radiotherapy for the Comprehensive Treatment of Oligometastatic Disease trial deserves mention as well. ${ }^{15,22}$ Although this study enrolled patients with multiple primary tumor types, including breast, colorectal, prostate, and NSCLC, stage IV NSCLC was one of the most common primary tumor types in this trial, constituting $18 \%$ of the total. Patients with controlled primary tumors with 5 or less metastatic lesions were eligible to enroll in this phase II, multicenter, randomized controlled trial comparing SABR plus standard-of-care (SOC) treatment to palliative SOC (palliative chemotherapy or radiation). Choice of systemic therapy in each arm was left to the treating medical oncologist. A total of 99 patients were enrolled: 66 in the SABR-plus-SOC arm and 33 in the palliative-SOC arm. Long-term follow-up demonstrated an impressive 5year OS of $42 \%$ (95\% CI, 28-56) in the SABR-plus-SOC arm versus $18 \%(95 \% \mathrm{CI}, 6-34 ; P=.006)$ in the palliative-SOC arm. Five-year PFS was $17 \%(95 \%$ CI, 8 -30) in the SABR-plus-SOC arm versus not reached in the palliative-SOC arm $(3.2 \%$ [95\% CI, 0-14] at 4 years, with the last patient censored; $P=.001) .{ }^{22}$ Notably, there were 3 treatment-related deaths in the SABR arm. Despite the excellent OS achieved in this trial, the relatively high treatment-related mortality $(4.5 \%)$ and the inclusion of mixed tumor types, some of which tend to have more prolonged survival in the metastatic state (eg, breast and prostate) versus other primary sites, make it difficult to draw definitive conclusions. ${ }^{23}$ Furthermore, the randomization procedure did not stratify by primary tumor type, ultimately leading to a control arm with only $21 \%$ breast and prostate cancers versus $41 \%$ in the SABR arm, which may have skewed the results.

On the basis of this growing body of evidence, multiple international guideline committees recommend LCT for select patients with oligometastatic NSCLC. ${ }^{24,25}$ The European Society of Medical Oncology guidelines for metastatic NSCLC recommend radical local treatment (surgery or high-dose radiotherapy) for oligometastatic disease. National Comprehensive Cancer Network guidelines for NSCLC recommend surgical resection, SABR, or definitive radiotherapy for the management of oligometastatic disease. Data are limited for the use of image-guided thermal ablation (eg, cryotherapy, microwave, or radiofrequency) techniques for oligometastatic NSCLC. ${ }^{26,27}$ The National Comprehensive Cancer Network (NSCL-15 guideline, version 3.2021) states that thermal ablation "may be an option for select patients not receiving radiotherapy or surgery." 24

Although we have good evidence from a few small phase II randomized trials, much is still unknown regarding which specific patients with oligometastatic NSCLC may benefit from LCT. Further confirmatory randomized trials involving patients with limited metastatic NSCLC receiving LCT (surgery, radiotherapy, or radiofrequency ablation) are ongoing and are summarized in Table 3.

\section{SURGICAL CONSIDERATIONS}

Most of the literature evaluating the use of surgical resection for oligometastatic NSCLC is confined to retrospective case series. Accordingly, surgeons must extrapolate from the results of the SABR-dominated trials reviewed. Although some of these trials included surgical resection as a possible definitive treatment option, surgery was infrequently used.

Several factors should be considered when contemplating surgery or ablative techniques for oligometastatic disease (Table 4). Foremost, the postoperative quality of life of the patient should be balanced with their desire to achieve a disease-free state. Although cure is possible with limited metastases, patients should understand such cases are rare. As with any oncologic surgical procedure, medical operability and resectability (of all involved sites) must be critically ascertained; arguably, this standard 
TABLE 3. Ongoing randomized controlled trials for local therapy in oligometastatic disease

\begin{tabular}{llclcccc}
\hline \multicolumn{1}{c}{ Study } & Phase & $\begin{array}{c}\text { Metastases, } \\
\text { no. }\end{array}$ & Type of LCT & $\begin{array}{c}\text { Expected } \\
\text { accrual }\end{array}$ & $\begin{array}{c}\text { Solid tumor } \\
\text { type(s) }\end{array}$ & $\begin{array}{c}\text { End } \\
\text { point }\end{array}$ & $\begin{array}{c}\text { Projected } \\
\text { completion }\end{array}$ \\
\hline OMEGA & III & $1-3$ & $\begin{array}{c}\text { Surgery, } \\
\text { RT, or RFA }\end{array}$ & 195 & NSCLC & OS & September 2022 \\
NRG-LU002 & II/III & $1-3$ & RT & 400 & NSCLC & PFS/OS & August 2027 \\
SARON & III & $1-5$ & RT & 340 & NSCLC & OS & August 2022 \\
CORE & II/III & $1-3$ & RT & 206 & Breast, NSCLC, & PFS & October 2024 \\
& & & & prostate & & \\
\hline
\end{tabular}

$\overline{L C T}$, Local consolidative therapy; $R T$, radiotherapy; RFA, radiofrequency ablation; $N S C L C$, non-small cell lung cancer; $O S$, overall survival; $P F S$, progression-free survival.

becomes even more important in patients with stage IV disease, in whom survival is limited. If complete resectability is uncertain, we favor nonsurgical strategies. Whether treatment should be tailored to the type of oligometastatic disease (eg, synchronous, metastatic, oligoprogressive) is not clear and a subject of active investigation. ${ }^{6,25}$

A thorough staging assessment in accordance with current guidelines must be undertaken, including brain magnetic resonance imaging (or head computed tomography scan) and whole-body positron emission tomography scan. ${ }^{24}$ Although data are inconsistent across studies, most suggest nodal status is associated with OS and PFS after LCT. Therefore, we believe mediastinal nodal staging with endobronchial ultrasound or mediastinoscopy is mandatory before proceeding with LCT for synchronous or metachronous oligometastatic disease. In principle, we do not offer LCT to metastatic patients with $\mathrm{N} 2$ or N3 disease.

In cases with more than 1 metastatic site involving different body cavities, a combined surgical and ablative approach using SABR, SRS, or percutaneous imageguided ablation techniques should be considered, especially when complete resection is questionable. Multiple metastases within a single pulmonary lobe are often best addressed with lobectomy, when appropriate. Thoracic surgeons commonly see patients with multiple histologically similar lung cancers. Tumor genotyping can be used to determine whether these tumors represent independent primaries or metastases. $^{28}$

All patients with metastatic NSCLC should undergo tumor genotyping to identify potential targeted therapy options. Patients with oligometastases with oncogenic driver alterations who are matched to appropriate therapy and undergo LCT to their primary tumors and metastases have better survival than similar patients without such driver mutations. ${ }^{13}$ The optimal sequence of systemic therapy and LCT has not been fully elucidated. For patients with more than 1 extracranial metastasis, current evidence supports starting with systemic therapy (to achieve control of systemic micrometastatic disease) and then proceeding with LCT, provided there is no disease progression. For patients receiving targeted or immune therapies who initially experience a good response but later develop limited sites of progression $(\leq 5)$, we and others generally recommend reinitiating these agents after LCT. ${ }^{29,30}$ Recently, the use of plasma circulating tumor DNA analysis has shown promise as a predictive biomarker for lung cancer treatment; further research using ultrasensitive assays for minimal residual disease is needed in the oligometastatic setting. ${ }^{31,32}$

TABLE 4. Recommended selection criteria for surgical resection or ablation of oligometastatic non-small cell lung cancer

Selection criteria

- $\leq 5$ metastatic sites

- Preoperative/preablation biopsy should be considered mandatory to confirm presence of metastatic disease before performing $\geq$ lobectomy and is strongly preferred in all other cases.

- In cases where it is unclear whether the lesion in question represents a new primary or a metastasis, expert histopathologic review and tumoral genotyping are recommended.

- All metastatic sites must be addressable with surgery (ie, complete R0 resection), ablation, or a combined modality approach.

- Mandatory FDG-PET and brain MRI (or CT head) obtained within $30 \mathrm{~d}$ of procedure

- Mandatory pathologic mediastinal nodal assessment (regardless of PET and CT findings) with mediastinoscopy or EBUS, with no evidence of N2 or N3 nodal involvement

- For patients with $>1$ extracranial metastasis, starting with a course of systemic therapy is favored. If the patient experiences progression of disease beyond the original metastatic sites while on systemic therapy, LCT is generally not offered.

- Multidisciplinary tumor board discussion and consensus

FDG-PET, (18) F-fluorodeoxyglucose positron emission tomography; MRI, magnetic resonance imaging; $C T$, computed tomography; PET, positron emission tomography; EBUS, endobronchial ultrasound; $L C T$, local consolidative therapy. 


\section{CONCLUSIONS}

Carefully selected patients with oligometastatic NSCLC will benefit from a combination of local surgical or ablative approaches and systemic therapy. Currently, data specific to the surgical treatment of oligometastatic NSCLC from randomized studies are lacking. While we await the results of ongoing large randomized trials, including OMEGA (NCT03827577), NRG-LU002 (NCT03137771), and SORAN (NCT02417662), we must base our treatment algorithms on high-level evidence. Key prognostic factors frequently associated with improved OS in patients with oligometastatic disease include control of the primary tumor, fewer metastatic sites $(\leq 5)$, metachronous over synchronous metastases, lower nodal stage $(\mathrm{N} 0>\mathrm{N} 1>\mathrm{N} 2>\mathrm{N} 3)$, response to first-line systemic therapy, and a longer disease-free interval ( $\geq 6$ months). We and others are investigating predictive biomarkers, such as circulating tumor DNA, to further guide precision therapy for oligometastatic NSCLC. ${ }^{31}$ In light of expanding immune and targeted therapy options, as well as the proven benefits of LCT in select cases, the former tendency toward treatment nihilism in oligometastatic NSCLC must be avoided. By use of a multidisciplinary approach and careful selection of the appropriate combination of available therapies, patients with oligometastatic NSCLC are now experiencing improved survival and a better quality of life.

\section{Conflict of Interest Statement}

J.M.I. has equity in LumaCyte LLC, serves as an uncompensated consultant to Roche-Genentech, and has received institutional research support from Grail and Guardant Health. B.T.L. has served as a consultant for ThermoFisher Scientific, Guardant Health, and Hengrui Therapeutics and an uncompensated consultant for Amgen, Lilly, Boehringer-Ingelheim, and Genentech. He has received institutional research funding from Amgen, Genentech, AstraZeneca, Daiichi-Sankyo, Lilly, Illumina, GRAIL, Guardant Health, Hengrui Therapeutics, MORE Health, Bolt Therapeutics, and BioMed Valley Discoveries. He has received academic travel support from Resolution Bioscience, MORE Health, and Jiangsu Hengrui Medicine. He is an inventor on 2 institutional patents at MSK and has intellectual property rights as a book author at Karger Publishers. D.R.G. received honoraria from Varian, BMS, Vindico, Medscape, and AstraZeneca, and research funding from Merck, AstraZeneca, and BMS.

The Journal policy requires editors and reviewers to disclose conflicts of interest and to decline handling or reviewing manuscripts for which they may have a conflict of interest. The editors and reviewers of this article have no conflicts of interest.
The authors thank David B. Sewell of the Department of Surgery, Memorial Sloan Kettering Cancer Center, for his superb editorial assistance in preparing this manuscript.

\section{References}

1. Ashworth A, Rodrigues G, Boldt G, Palma D. Is there an oligometastatic state in non-small cell lung cancer? A systematic review of the literature. Lung Cancer. 2013;82:197-203.

2. Ashworth AB, Senan S, Palma DA, Riquet M, Ahn YC, Ricardi U, et al. An individual patient data metaanalysis of outcomes and prognostic factors after treatment of oligometastatic non-small-cell lung cancer. Clin Lung Cancer. 2014;15: 346-55.

3. Endo C, Hasumi T, Matsumura Y, Sato N, Deguchi H, Oizumi H, et al. A prospective study of surgical procedures for patients with oligometastatic non-small cell lung cancer. Ann Thorac Surg. 2014;98:258-64.

4. Hellman S, Weichselbaum RR. Oligometastases. J Clin Oncol. 1995;13:8-10.

5. Weichselbaum RR, Hellman S. Oligometastases revisited. Nat Rev Clin Oncol. 2011:8:378-82.

6. Guckenberger M, Lievens Y, Bouma AB, Collette L, Dekker A, deSouza NM, et al. Characterisation and classification of oligometastatic disease: a European Society for Radiotherapy and Oncology and European Organisation for Research and Treatment of Cancer consensus recommendation. Lancet Oncol. 2020;21:e18-28.

7. Lievens Y, Guckenberger M, Gomez D, Hoyer M, Iyengar P, Kindts I, et al. Defining oligometastatic disease from a radiation oncology perspective: an ESTRO-ASTRO consensus document. Radiother Oncol. 2020;148:157-66.

8. Rusthoven KE, Hammerman SF, Kavanagh BD, Birtwhistle MJ, Stares M, Camidge DR. Is there a role for consolidative stereotactic body radiation therapy following first-line systemic therapy for metastatic lung cancer? A patterns-offailure analysis. Acta Oncol. 2009;48:578-83.

9. Eberhardt WE, Mitchell A, Crowley J, Kondo H, Kim YT, Turrisi A III, et al. The IASLC Lung Cancer Staging Project: proposals for the revision of the M descriptors in the forthcoming eighth edition of the TNM classification of lung cancer. $J$ Thorac Oncol. 2015;10:1515-22.

10. American Joint Committee on Cancer, American Cancer Society. AJCC Cancer Staging Manual. $8^{\text {th }}$ ed. Amin MB, Edge SB, Gress DM, Meyer LR, eds. Chicago, IL: American Joint Committee on Cancer, Springer; 2017.

11. Downey RJ, Ng KK, Kris MG, Bains MS, Miller VA, Heelan R, et al. A phase II trial of chemotherapy and surgery for non-small cell lung cancer patients with a synchronous solitary metastasis. Lung Cancer. 2002;38:193-7.

12. Gomez DR, Blumenschein GR, Lee JJ, Hernandez M, Ye R, Camidge DR, et al. Local consolidative therapy versus maintenance therapy or observation for patients with oligometastatic non-small-cell lung cancer without progression after first-line systemic therapy: a multicentre, randomised, controlled, phase 2 study Lancet Oncol. 2016;17:1672-82.

13. Gomez DR, Tang C, Zhang J, Blumenschein GR, Hernandez M, Jack Lee J, et al. Local consolidative therapy vs maintenance therapy or observation for patients with oligometastatic non-small-cell lung cancer: long-term results of a multiinstitutional, phase II, randomized study. J Clin Oncol. 2019;37:1558-65.

14. Iyengar P, Wardak Z, Gerber DE, Tumati V, Ahn C, Hughes RS, et al. Consolidative radiotherapy for limited metastatic non-small-cell lung cancer: a phase 2 randomized clinical trial. JAMA Oncol. 2018;4:e173501.

15. Palma DA, Olson R, Harrow S, Gaede S, Louie AV, Haasbeek C, et al. Stereotactic ablative radiotherapy versus standard of care palliative treatment in patients with oligometastatic cancers (SABR-COMET): a randomised, phase 2, openlabel trial. Lancet. 2019;393:2051-8.

16. Congedo MT, Cesario A, Lococo F, De Waure C, Apolone G, Meacci E, et al. Surgery for oligometastatic non-small cell lung cancer: long-term results from a single center experience. J Thorac Cardiovasc Surg. 2012;144:444-52.

17. Tonnies M, Pfannschmidt J, Bauer TT, Kollmeier J, Tonnies S, Kaiser D. Metastectomy for synchronous solitary non-small cell lung cancer metastases. Ann Thorac Surg. 2014;98:249-56.

18. Ngwa W, Irabor OC, Schoenfeld JD, Hesser J, Demaria S, Formenti SC. Using immunotherapy to boost the abscopal effect. Nat Rev Cancer. 2018;18:313-22.

19. Cushman TR, Gomez D, Kumar R, Likacheva A, Chang JY, Cadena AP, et al Combining radiation plus immunotherapy to improve systemic immune response. J Thorac Dis. 2018;10:S468-79.

20. Theelen WS, de Jong MC, Baas P. Synergizing systemic responses by combining immunotherapy with radiotherapy in metastatic non-small cell lung cancer: the potential of the abscopal effect. Lung Cancer. 2020;142:106-13. 
21. De Ruysscher D, Wanders R, Hendriks LE, van Baardwijk A, Reymen B, Houben R, et al. Progression-free survival and overall survival beyond 5 years of NSCLC patients with synchronous oligometastases treated in a prospective phase II trial (NCT 01282450). J Thorac Oncol. 2018;13:1958-61.

22. Palma DA, Olson R, Harrow S, Gaede S, Louie AV, Haasbeek C, et al. Stereotactic ablative radiotherapy for the comprehensive treatment of oligometastatic cancers: long-term results of the SABR-COMET phase II randomized trial. J Clin Oncol. 2020;38:2830-8.

23. Kamarinos NV, Dawson LA, Saltz LB, Crane CH, Overman MJ, Vauthey JN, et al. Trials of locoregional therapies inspired by SABR-COMET. Lancet. 2020;396:956-7.

24. National Comprehensive Cancer Network. NCCN Guidelines: Non-Small Cell Lung Cancer. Version 8. NCCN; 2021. Available at: https://www.ncen.org/ professionals/physician_gls/pdf/nscl.pdf. Accessed March 1, 2021.

25. Planchard D, Popat S, Kerr K, Novello S, Smit EF, Faivre-Finn C, et al. Metastatic non-small cell lung cancer: ESMO clinical practice guidelines for diagnosis, treatment and follow-up. Ann Oncol. 2018;29:iv192-237.

26. Wei Z, Ye X, Yang X, Huang G, Li W, Han X, et al. Efficacy and safety of microwave ablation in the treatment of patients with oligometastatic nonsmall-cell lung cancer: a retrospective study. Int J Hyperthermia. 2019;36: 827-34.

27. Ni Y, Ye X, Yang X, Huang G, Li W, Wang J, et al. Microwave ablation as local consolidative therapy for patients with extracranial oligometastatic EGFR- mutant non-small cell lung cancer without progression after first-line EGFRTKIs treatment. J Cancer Res Clin Oncol. 2020;146:197-203.

28. Girard N, Ostrovnaya I, Lau C, Park B, Ladanyi M, Finley D, et al. Genomic and mutational profiling to assess clonal relationships between multiple non-small cell lung cancers. Clin Cancer Res. 2009;15:5184-90.

29. Yang JJ, Chen HJ, Yan HH, Zhang XC, Zhou Q, Su J, et al. Clinical modes of EGFR tyrosine kinase inhibitor failure and subsequent management in advanced non-small cell lung cancer. Lung Cancer. 2013;79:33-9.

30. Yu HA, Sima CS, Huang J, Solomon SB, Rimner A, Paik P, et al. Loca therapy with continued EGFR tyrosine kinase inhibitor therapy as a treatment strategy in EGFR-mutant advanced lung cancers that have developed acquired resistance to EGFR tyrosine kinase inhibitors. J Thorac Oncol. 2013;8 346-51.

31. Isbell JM, Jones DR, Li BT. Circulating tumor DNA: a promising biomarker to guide postoperative treatment and surveillance of non-small cell lung cancer. $J$ Thorac Cardiovasc Surg. 2018;155:2628-31.

32. Moding EJ, Diehn M, Wakelee HA. Circulating tumor DNA testing in advanced non-small cell lung cancer. Lung Cancer. 2018;119:42-7.

Key Words: local therapy, non-small cell lung cancer, oligometastasis 